\title{
2. Law as Thirdness
}

By NOW, THE READER ought not be surprised to learn that Peirce's theory of "law" is intimately connected with his universal categories. It has already become clear that he situates law in the category of Thirdness and that Thirdness involves generality or continuity. We propose to examine in detail the characteristics of this category as Peirce applied them to "real laws of nature." Again, however, we must realize that we can hardly expect to find absolute consistency in the formulations employed over so many years in such prolific writing for such varied occasions. At best, we can hope to find a clear and firm line of thought-a uniform direction in which Peirce's whole philosophic thinking is developing. With these cautions in mind let us begin by examining the chief characteristics ascribed to Thirdness and hence to law in order to see how they are interrelated.

Fundamentally Thirdness is mediation. It is a medium between Firstness and Secondness. In terms of Peirce's modes of being, then, law mediates between pure possibility and actual fact. But in order so to mediate it must be general; it must be neither of the extremes and yet partake of aspects of both. Finally, because of its generality Thirdness must essentially refer to the future.

First, Thirdness is mediation. ${ }^{1}$ Consider Peirce's homely but apt illustration of the cook who desires to make an apple pie for her master. The apple pie she desires is no particular one, but only one of a certain kind, of a certain general description. She has an idea of the sort of pie she desires and this idea taken in itself, independently of the deliberate decision to take the necessary steps to produce a concrete singular instance of that type of pie, is a pure possible-the airy object of a dream. Between the dream and the finished product is the efficacious desire. Again, to make the pie she has to pick out some

1 "By the third, I mean the medium or connecting bond between the absolute first and last. . . . Continuity represents Thirdness almost to perfection" (1.337 from a fragment, "Third," ca. 1875). Peirce came to this notion early in his career. 
apples. Any good apples will do although she cannot pick out "any apple" but this one or that. It is her desire to select apples of a certain type which mediates between the given quality and the concrete case.

What she desires is something of a given quality; what she has to take is this or that particular apple. From the nature of things, she cannot take the quality but must take the particular thing. Sensation and volition being affairs of action and reaction relate to particular things. She has seen only particular apples. But the desire has nothing to do with particulars; it relates to qualities. Desire is not a reaction with reference to a particular thing; it is an idea about an idea, namely, the idea of how delightful it would be for me, the cook's master, to eat an apple pie. (1.341)

The object of the desire is not an unattached quality.

She [the cook] has no particular apple pie she particularly prefers to serve; but she does desire and intend to serve an apple pie to a particular person. ... Throughout her whole proceedings she pursues an idea or dream without any particular thisness or thatness-or, as we say, hecceity - to it, but this dream she wishes to realize in connection with an object of experience, which as such, does possess hecceity; and since she has to act, and action only relates to this and that, she has to be perpetually making random selections, that is, taking whatever comes handiest. (1.341)

Peirce explicitly spells out the moral of the story.

The dream itself has no prominent thirdness; it is, on the contrary, utterly irresponsible; it is whatever it pleases. The object of experience as a reality is a second. But the desire in seeking to attach the one to the other is a third, or medium. (1.342)

It illustrates what Peirce understands by mediation and why mediation always involves a Third. Yet it must not be taken as a rigorous analysis, because it turns out that, more strictly speaking, representa- 
tion and not desire is the prime analogue of mediation. ${ }^{2}$ All that interests us for the moment is Peirce's generalization of the principle involved and its direct application to laws of nature.

So it is with any law of nature. Were it but a mere idea unrealized-and it is of the nature of an idea-it would be a pure first. The cases to which it applies, are seconds. (1.342)

But that condition is contrary to fact. Precisely because it applies to Seconds, a law of nature is not a pure First. It is then a Third or medium, and consequently belongs to the category of Thirdness since "Thirdness is nothing but the character of an object which embodies Betweenness or Mediation in its simplest and most rudimentary form ..." (5.104).

Second, mediation supposes generality. In the illustration of the cook and the pie we have seen that generality is involved in her desire. She desired to make some particular instance of a general kind. Furthermore all her actions in the actual process of baking, for example selecting the apples, were governed by general rules of conduct. But let us see if we can go a little deeper into the reason why mediation necessarily involves generality. Just what is required that anything at all be a medium? It must be distinct from what it mediates, although not necessarily separable. It is a Third, not a First nor a Second. Yet it must partake of the natures of what it brings together. It must be like a First and like a Second, that is, it must be both a First and not a First, and both a Second and not a Second. ${ }^{3}$ In other words, a

2 See Appendix II concerning the final logical interpretant.

${ }^{3}$ For Peirce vagueness and generality are two kinds of indeterminacy. The former is the antithetical analogue of the latter. A sign is objectively general, if it leaves it to the interpreter to supply further determinations. A sign is objectively vague, if it reserves for some other possible sign and not for the interpreter, the function of completing the determination. Thus, in the sentence "Man is mortal" the term "man" is objectively general because the answer to the question "What man?" is "Any one at all whom you may choose." But in the sentence, "This month a great event will happen," the term "a great event" is objectively vague because the answer to the question "What event?" is not "Any one you like," but rather "Let us wait and see." Thus it is that the principle of excluded middle does not apply to the 
medium is essentially indeterminate $(5.447-449,5.505)$. It is both vague and general, although not in the same respect (5.506). It is vague because in one sense the principle of contradiction does not apply to it and it is general because in another sense the principle of excluded middle does not apply to it (5.505). ${ }^{4}$ Thus the cook's desire is indeterminate with respect to her dream on the one hand and the product of her industry on the other. In terms of logic her desire is of the nature of a sign. Indeed Peirce sometimes says that mediation "reaches its fullness in Representation" (5.104), or that law is a matter of thought or meaning in things (1.343), or that law is of the nature of a general sign $(1.27,1.27 \mathrm{n} .1,1.26,5.107)$. In terms of mathematics, the essential indeterminateness of a medium, its vagueness and generality, is nothing but continuity. Hence he tells us that "true generality is . . . nothing but a rudimentary form of true continuity" (6.172). A point on a line, for example, could not connect the portions of that line if it were truly discrete and atomic. In that case it would be a break in the line. It can be thought of as joining the parts of the line only if it is continuous with its immediate predecessor and im-

general, while the principle of contradiction does not apply to the vague (5.505). No sign can be both vague and general at one and the same time and in one and the same respect, "since insofar as the right of determination is not distinctly extended to the interpreter it remains the right of the utterer" (5.506). The only way a sign can escape being either general or vague is by not being indeterminate, that is, by being both singular and definite. While a general predicate renders the singular subject of which it is predicated determinate with respect to itself, it leaves that subject indeterminate with regard to other predicates and remains itself indeterminate with regard to other subjects. A vague predicate does not determine its singular subject with regard to itself and $a$ fortiori is not itself determined to that subject. A vague predicate remains indefinite with respect to the subject. For Peirce, no communication between persons can be entirely definite. Wherever degree or any other possible continuous variation subsists no absolute precision is possible. And since no man's experience is exactly the same as another's, his interpretation of a word must be to some degree imprecise (5.506).

${ }^{4}$ Cf. M. Thompson, op. cit., pp. 213-218, for discussion of Peirce's analysis of vagueness. In two unpublished papers (1903 and 1909 respectively) Peirce explains that the principle of contradiction does not apply to "may-be's" or possibility and that the principle of excluded middle does not apply to "would-be's" or laws (Peirce Papers, \#641, pp. 24 4/5-24 5/6; \#642, pp. 20-22). 
mediate successor. This is the idea one tries to express by saying that a point has no length or breadth but only position. Or again, colors in a spectrum are continuous. There are no sharp gaps. The borderlines are assigned arbitrarily because in themselves the colors gradually blend one into the other so that at places on the scale it is impossible to say objectively whether the color is orange or red; perhaps it is both or neither.

Mediation, then, implies on Peirce's analysis both vagueness and generality. The continuous involves both these sorts of indeterminateness. Therefore, by applying the ultrarealistic principle that all logical distinctions are also metaphysical, it follows that real mediation implies real vagueness and real generality. Continuity is ingredient in reality.

Third, and finally, generality necessarily refers to the future. To understand what this assertion means we will have to consider in some detail the kind of generality Peirce ascribes to quality and the kind he ascribes to law. At first sight it may seem strange that Peirce should ascribe any generality at all to quality since he usually puts quality in the first category and not in the third. Indeed, it might be argued that here Peirce collapses Firstness into Thirdness. We think, however, that such an objection is based on a misunderstanding of the interdependence of Peirce's categories and a failure to distinguish between at least two ways in which he uses the term "quality."

Peirce does ascribe a certain generality to quality as well as to law. Thus in the "Logic of Mathematics" (ca. 1896) he says that qualities "merge into one another" (1.418) and thus exhibit a certain continuity. Again he says that qualities are somewhat vague and potential in contrast to occurrences which are perfectly individual. And that is why qualities do not make up facts although they are "concerned in" them (1.419). Or again, explaining what a general fact might be, he attributes its generality to its connection with the potential world of quality (1.420). The thing to note here is that quality is a "mere abstract potentiality" or possibility (1.422). Some have taken the position that Peirce is talking about logical possibility instead of real power. ${ }^{5}$ This is certainly a mistake. In the text Peirce criticizes those

5 E.g. Thomas A. Goudge, "The Views of Charles Peirce on the Given in Experience," Journal of Philosophy, XXXII (1935), 533-544. Cf. John Dewey's refutation, "Peirce's Theory of Quality," ibid., 701-708, reprinted in 
who claim that a thing does not have the quality of red in the dark, or that a piece of iron is not hard except when actually resisting pressure. Of course the quality of hardness or of redness is actualized only when the thing that has it interacts with something else, but before that interaction the thing really has that quality potentially, as a real power and not just as a logical possibility. Peirce describes quality as what "might happen" given the suitable conditions. It is a way of behaving and consequently it is understandable why Peirce sees it as general. It would be a nominalistic error to deny that things really have ways of behaving, that is, to deny that they have real potentialities. Yet this is the position to which one is forced who would make qualities merely logical possibilities.

Now the question is whether Peirce thought that quality, in the sense of potentiality, fits the category of Firstness. The answer is no. We have already cited in the previous section the text which makes this conclusion inescapable. It will be well to repeat it here.

A Firstness is exemplified in every quality of a total feeling. It is perfectly simple and without parts; and everything has its quality. Thus the tragedy of King Lear has its Firstness, its flavor sui generis. That wherein all such qualities agree is universal Firstness, the very being of Firstness. The word possibility fits it, except that possibility implies a relation to what exists, while universal Firstness is the mode of being of itself. That is why a new word was required for it. Otherwise, "possibility" would have answered the purpose. (1.531)

Possibility or potentiality, therefore, almost fits Firstness as such, but not quite. Consequently quality considered as potentiality does not quite fit Firstness either, because as potentiality quality implies a relation to Secondness or existence. Still, it is clear that Peirce does use quality to exemplify Firstness. Firstness is the "sheer totality and pervading unity of quality in everything experienced, whether it be odor, the drama of King Lear, or philosophic or scientific systems." ${ }^{\text {Peirce, }}$

R. Bernstein's John Dewey on Experience, Nature and Freedom (New York: Liberal Arts Press, 1960), pp. 199-210. I am much indebted to Dewey's article for this section.

6 Dewey, art. cit., p. 200, in Bernstein's edition. 
however, uses the term "quality" in two senses: as it is in itself, sheer totality and pervading unity, and in its relation to other aspects of a phenomenon, and in particular, to Secondness or existence. In this second sense, quality is not pure Firstness, precisely because it involves a relation to Secondness. More exactly, quality here is the Firstness of Secondness, and the co-presence of these two categories yields the generality necessary for potentiality or Thirdness. Quality per se, that is, in the first sense distinguished above, is pure Firstness, but as such is neither general nor individual. It is something like the scholastic natura communis, an abstract nature which is neither formally universal nor individuated. Or again it is like the comprehension of a term taken without any reference to its extension. In this sense, then, quality does not signify potentiality or possibility and there is some question in Peirce's mind whether quality per se can be conceived at all. He seems to say, as we shall see in the next paragraph, that it can only be pointed to by an indexical sign. Secondness per se is not general either. It is individual and even antigeneral $(7.132)^{7}$ in that it resists generalization to the point where it would lose its character as Secondness if it were generalized. In this, Secondness per se differs from quality per se or pure Firstness. Quality per se does not resist generalization. In fact, it is what makes generality possible at all. Hence, strictly speaking, Secondnesses taken in themselves have no common quality (1.532). Each Secondness is unique. Nevertheless Secondnesses as we experience them do have common qualities precisely because, as we experience them, they are inseparably bound up with Firstnesses. It follows, too, of course, that we can never experience pure Firstnesses or qualities per se either. We can experience them only in relation to Secondnesses, that is to say, only as real potentialities. Our human experience always involves this Thirdness, mediation, lawfulness. Indeed, Peirce defines Thirdness as

. . . that mode of being which consists, mind my word if you please, the mode of being which consists in the fact that future facts of Secondness will take on a determinate general character [Firstness]. (1.26)

7 By "anti-general" is meant that Secondness cannot be generalized after the manner of a law without losing its character of Secondness. Firstness may be so generalized without destruction of its character. 
It is no wonder, then, that Peirce finds no difficulty in assimilating qualities considered as potentialities to law expressible in conditionals.

The point to remember here is that Peirce's categories are never experienced in their purity. And we saw in our discussion of the categories in connection with the normative sciences, Firstness, Secondness, and Thirdness are distinguished as elements in every experience by precisive abstraction. Certainly, they are irreducible elements into which an experience must be analysed, but they are nonetheless inseparable. Thus, it will be recalled, Peirce warned us that it is very difficult so to distinguish them as to hold them each in their purity and yet in their full meaning (1.353). In the same paragraph he says that the categories are so intangible that they are tints or tones upon conceptions rather than conceptions. In yet another place, Peirce points out that the categories, "being enormously large, very promiscuous, and known but in small part, cannot be satisfactorily defined, and therefore can only be denoted by Indices" (4.544). The categorial structure which Peirce uses is therefore highly subtle and complex, admitting of various combinations. The more familiar terms which Peirce applies to his categories, therefore, must be approached with caution. They do not always fit exactly (e.g. "quality" and "possibility" for Firstness). ${ }^{8}$ Indeed no term describing our experience could exactly capture just one of the categories since our experience will always involve all three categories. At least this will be true of any experience which we are consciously examining. By examining it, we are knowing it. By knowing it we are judging and interpreting it. Whatever is known, insofar as it is known, has a share of Thirdness. But Thirdness is logically dependent upon Firstness and Secondness.

Thus Peirce himself distinguishes qualities in themselves and qualities as reflected upon.

When we say that qualities are general, are partial determinations, are mere potentialities, etc., all that is true

8 Peirce distinguished the matter of phenomena from the forms of experience. The categories of the former are quality, fact, and law, while the categories of the latter are the monad, the dyad, and the polyad. Both sets of categories are under Firstness, Secondness, and Thirdness as universal categories (see 1.452). 
of qualities reflected upon; but these things do not belong to the quality-element of experience. (1.425)

A quality reflected upon is one asserted of a subject in a judgment. It is referred to some other aspect of a phenomenon and so takes on generality. But in itself, taken in abstraction, "the quality is what presents itself in the monadic aspect" of experience (1.424).

The phenomenon may be ever so complex and heterogeneous. That circumstance will make no particular difference in the quality. It will make it more general.

But one quality is in itself, in its monadic aspect, no more general than another. The resultant effect has no parts. The quality in itself is indecomposable and sui generis. (1.425)

Notice that the monadic aspect of experience is not itself experienced. It is gotten from experience through precisive abstraction. This is the "quality-element" of experience.

In a later fragment ( $c a .1904)$ Peirce characterized these qualities in themselves as mere "may-be's" which are not necessarily realized. Their being consists in the fact that there might be such a peculiar, positive, suchness in a "phaneron." And to emphasize that these qualities in themselves do not imply any relation to Secondness or existence, he adds that they are merely a question of what one can imagine (e.g. a being whose whole life "should consist in nothing at all but a violet color or a stink of cabbage") and not of what psychological laws permit. This very fact

... shows that such a feeling is not general, in the sense in which the law of gravitation is general. For nobody can imagine that law to have any being of any kind if it were impossible that there should exist two masses of matter, or if there were no such thing as motion. A true general cannot have any being unless there is to be some prospect of its sometime having occasion to be embodied in a fact, which is itself not a law or anything like a law. A quality of feeling can be imagined to be without any occurrence, as it seems to me. Its mere may-being gets along without any realization at all. (1.304, emphasis added)

Potentiality, or quality as reflected upon, and law, therefore, are "true 
generals" in Peirce's sense. Now since the very notion of potentiality refers to the future (what can or could be) and since only something which is or involves potentiality is a true general, we have established the proposition that generality necessarily refers to the future.

And yet we are not quite finished. It remains to discuss the relation between potentiality and law, for Peirce explicitly distinguishes the sort of "true generality" proper to each. In the Logic of Mathematics, explaining what must be excluded from the category of fact or Secondness, Peirce writes,

This is the general, and with it the permanent or eternal (for permanence is a species of generality), and the conditional (which equally involves generality). Generality is either of that negative sort which belongs to the merely potential, as such, and this is peculiar to the category of quality; or it is of that positive kind which belongs to conditional necessity, and this is peculiar to the category of law. (1.427)

Peirce never explains in so many words what he means by negative and positive generality. He only gives us hints and leaves us to puzzle it out. The clue he gives us is a set of contrasts: (1) the permanent or eternal as opposed to the conditional, (2) the potential in contrast to the conditionally necessary, (3) quality compared with law. Presumably, then, negative generality is that generality which characterizes quality as potentiality, and potentiality has something permanent or eternal about it. The permanent or eternal is what is time-independent. It holds good always, under any circumstances. It would seem, therefore, to be an a priori condition or formal law. Did we not just say, however, that the potential necessarily refers to the future? Is it not, therefore, time-dependent and mutable? The way out of this dilemma can be found by examining more closely how we express potentiality. Consider Peirce's own famous example of "hardness." The test of a material's hardness is scratching. But no one would want to say that the testing constituted the hardness. The material was hard antecedent to the test (even though perhaps we did not know it until the test was made). Furthermore, a different specimen of the same sort of material would still be judged hard even if no test were ever made upon it. Therefore, we express real potentiality in terms 
of subjunctive conditionals: "If such and such were rubbed with this material, it would resist marking." This conditional might be a contrary-to-fact, yet it would still be true. In one sense then it is permanent, eternal, time-independent. The conditional does not specify any particular time or time-interval $t$. And yet the conditional does in a general way refer to the future. It says that would happen, if a certain set of conditions were fulfilled. This sort of conditional proposition does not assert the antecedent or the consequent (neither as contingent nor as necessary); it asserts the connection between them or the consequence as necessary. The Universe of this sort of proposition is that of possibility. It is therefore independent of the actual timeorder, but not independent of a possible time-order. ${ }^{9}$ It should be clear, however, that what the subjunctive conditional asserts as true gives no direct information concerning how one knows that it is true, nor does the assertion qua assertion need to furnish the evidence for its truth. The point is that while the assertion of real potentiality concerns the Universe of Possibility, the evidence for the truth of that assertion must be obtained in and through the Universe of Actuality by means of induction. ${ }^{10}$

Thus potentiality is in a sense permanent or eternal in that it expresses a necessary relationship. The necessity, however, is not unconditional. Unconditional necessity would attach to the antecedent or to the consequent either as brute fact which happened once and for all in the irretrievable past, or as brute force-force without law or reason (1.427). The necessity is conditional or relational, and has permanence or eternality as a relationship. Of course, if the conditions of the relation are altered one has another and different relation. The former does not ipso facto cease to be a real possibility (at the very

9 In "Prolegomena to an Apology for Pragmaticism" (Monist, 16 [1906]), Peirce identifies the modes of being (actuality, possibility, destiny) with the universes, not with his categories. "On the contrary," he says, "the succession of Predicates of Predicates is different in the different Modes of Being" (4.549). Each of the three universes must be divided into "realms" for the different predicaments or categories. Potentiality then would turn out to be the Thirdness of the universe of possibility.

10 For Peirce, what distinguishes a "true law" from mere "regularities" or summations of past experience is the way in which they are discovered (7.84). 
least a logical possibility). We see no objection to nor inconsistency in Peirce's regarding potentiality as a kind of law.

Still Peirce distinguished the sort of generality proper to law, positive generality, from that proper to potentiality, negative generality. Consequently, he does not simply identify "law" and "potentiality." Indeed, Peirce's usual formulation of what a law is, is not in terms of the "would-be" of potentiality but in those of the "will-be" of actuality. To be sure, law in this more usual sense is expressed in a conditional, but that conditional is usually in the indicative mood. It takes the form of a prediction. "If such and such material is rubbed, it will resist marking."

A law of nature, then, will be regarded as having a sort of esse in futuro. That is to say they will have a present reality which consists in the fact that events will happen according to the formulation of those laws. (5.48)

Again, enumerating the three modes of being, Peirce describes the being of law as that which "will govern facts in the future" (1.23). In another place, he writes:

When an experimentalist speaks of phenomenon ... he does not mean any particular event that did happen to somebody in the dead past, but what surely will happen to everybody in the living future who shall fulfill certain conditions. (5.425)

In yet another context, Peirce describes law as "how an endless future must continue to be" (1.534), stressing the necessity, albeit conditional, of a true law.

In the light of these clear statements it is perhaps fair to say that the distinction between negative and positive generality is the same (or almost the same) as that which exists between "would-be" and "will-be," between the subjunctive and the indicative. The difference which the speaker intends to convey by his choice of mood is one of emphasis. The mood signals a point of view or point of interest to be taken into consideration in determining the sentence's meaning. It signals what we called before a Universe. ${ }^{11}$ Peirce's Universes are

11 A shift in the modal auxiliary indicates another change of emphasis, namely, a change of category. For example, a shift to "may-be" and "might- 
those which correspond to what he takes to be the basic modes of being. Both "would-be" and "will-be" are forms employed in future conditional sentences. The "would-be" emphasizes the necessary connection or the consequence, leaving out of consideration the factual status of antecedent and consequent. It stresses the relationship between kinds of antecedents and consequents, and only implies a relation to actual instances (as the basis for the generalization, let us say, in past experience). The "will-be" formulation, while including the necessary connection or consequence of the "would-be" as the very basis for the prediction, emphasizes the factual status of antecedent and consequent as about to be realized, probably to be realized, surely to be realized in the long run, etc. While it deals with kinds of facts and their relations, it adds an explicit reference to the actual instances in the order of our actual experience.

1) If $X$ were rubbed with $Z, X$ would resist marking by $Z$.

2) If $X$ is rubbed with $Z, X$ will resist marking by $Z$.

For Peirce, Sentence 2 is a confident prediction as to the actual occurrence of an actual fact in our experience on the condition that another actual occurrence of an actual fact be realized. The confidence of this prediction is based on the necessary connection or consequence expressed in Sentence 1, even though Sentence 1 gives no indication, expectation, or hope that $\mathrm{Z}$ will ever, as a matter of fact, touch $\mathrm{X}$. We suggest, therefore, that the distinction between negative and positive generality has to do with the absence or the presence in the formulation of explicit reference to the actual world of our experience. Potentiality, expressed in would-be's, the foundation of and necessary condition for law, expressed in will-be's (and, therefore, capable of being called a "law" by analogy), is only negatively general for Peirce because it consciously refrains from explicit reference to the factual status of its antecedent and consequent, while law is positively general because it consciously makes such a reference. From one point of view, then, "would-be" conditionals are stronger claims than "willbe's" since they are the basis for the prediction in the first place. Yet from another point of view they say less because they prescind from the actual occurrences in our world of experiences.

be" seems to indicate in Peirce a shift to Firstness as such, to quality in itself (1.304). 
We have indeed gone far afield in attempting to understand why Peirce conceived generality as necessarily implying a reference to the future. Whether we understand generality as negative or as positive our discussion has clearly shown that both indeed do so refer. Both potentiality and conditional necessity by definition imply such a reference, and so both are characterized by Thirdness. ${ }^{12}$ There are several other problems which must be explored (e.g. the distinction between formal and material laws, and the notion of destiny), but at this point a summary of what we have seen may be useful. So far in searching for an understanding of Peirce's use of the term "law," we have found that as essentially Thirdness or thought (1) it mediates between qualities in themselves (Firstnesses) and facts or actualities (Secondnesses), (2) it is therefore indeterminate, that is, vague and general, and so is a special case of continuity, and finally (3) its

12 "We may say that the bulk of what is actually done consists of Secondness-or better, Secondness is the predominant character of what has been done. The immediate present, could we seize it, would have no character but its Firstness. Nor that I mean to say that immediate consciousness (a pure fiction, by the way), would be Firstness, but that the quality of what we are immediately conscious of, which is no fiction, is Firstness. . . . [W] [Wat is to be, according to our conception of it, can never be wholly past. . . . I call this element of the phenomenon or object of thought the element of Thirdness. It is that which is what it is by virtue of imparting a quality to reactions in the future" (1.343).

Past as past is over and done with. It can never be retrieved. It is frozen as it were with all the singularity and concreteness of its happening. To this extent, then, Secondness is its specific characteristic. Still, this does not mean that there is nothing of Firstness and Thirdness in past events. Since everything and every event is what it is, past events have Firstness about thembut not insofar as they are past. Again, since we can and do generalize about past events and since every concrete, singular event is also an instance of a type, past events have Thirdness about them, but not qua past. Similarly, the present as present, the indivisible nunc, is only what it is with no reference to anything else. Its specific character is Firstness. Yet it manifests Secondness and Thirdness: secondness because any quality to be experienced must be embodied in an actual event; Thirdness because the present is embedded in a continuum. The future qua future is characterized by Thirdness, because it is indeterminate. It has reality only as potentiality really in actual things capable of future determination. But of course, future events will manifest Firstness and Secondness insofar as they are events, not insofar as they are future. 
specific sort of generality ("true generality") refers to the future. In short, law is founded in real potentiality in things, and can be best formulated in a conditional proposition. Here are some of the ways in which Peirce describes law. (Bracketed words have been inserted to indicate the presence of the three elements just enumerated.)

A law of nature, then, will be regarded ... as having a sort of esse in futuro. That is to say they will have a present reality [real potentiality] which consists in the fact that events [Secondnesses] will happen according to the formulation [a symbolic representation to which Firstnesses are essential] of those laws. (5.48)

In another place, and perhaps more clearly, he says:

[Thirdness is that mode of being] which consists, mind my word if you please, the mode of being which consists in the fact that future facts of Secondness will take on a determinate general character [Firstness].... (1.26)

Or again,

My view is that there are three modes of being. I hold that we can directly observe them in elements of whatever is at any time before the mind in any way. They are the being of positive qualitative possibility, the being of actual fact, and the being of law that will govern [hence mediate] facts [of a certain positive quality] in the future. (1.23)

In another place, Peirce underlines the conditional element in law.

When an experimentalist speaks of phenomenon ... he does not mean any particular event that did happen to somebody in the dead past [Secondness], but what surely will happen to every body in the living future who shall fulfill certain conditions. (5.425)

Again, in answering the objection that one cannot consistently hold both that to be and to be represented are not identical and that the nature of real law is to be represented (if the laws are real, they are 
not of the nature of representation; if they are of the nature of representation, they are not real), he writes,

My answer to this would be that it rests upon an ambiguity. When I say that the general proposition as to what will happen, whenever a certain condition may be fulfilled, is of the nature of a representation, I mean that it refers to experiences in futuro, which I do not know are all of them experienced and never can know have been all experienced. But when I say that really to be is different from being represented, I mean that what really is, ultimately consists in what shall be forced upon us in experience, that there is an element of brute compulsion in fact, and that fact is not a mere question of reasonableness. (5.97)

It is this reference to the future which distinguishes lawfulness from mere uniformity exhibited in past events. Rolling six straight passes with honest dice is a mere uniformity serving no basis for prediction concerning the seventh throw. Thus in a review of Herbert Nichol's A Treatise on Cosmology (ca. 1904) he criticizes John Stuart Mill for not having seen precisely this difference.

We all know that John Mill banished the word 'law' and substituted 'uniformity' for it, as more precisely expressing what it meant. But pragmatism discovers a serious error here. For while uniformity is a character which might be realized, in all its fulness, in a short series of past events, law, on the other hand, is essentially a character of an indefinite future; and while uniformity involves a regularity exact and exceptionless, law only requires an approach to uniformity in a decided majority of cases. (8.192)

Peirce explains that law could reasonably affect human conduct only through the knowledge of such a law creating and warranting anticipations of future experience. And what this requires is

... that the law should be a truth expressible as a conditional proposition whose antecedent and consequent express experiences in a future tense, and further, that, as long as the law retains the character of a law, there should be 
possible occasions in an indefinite future when events of the kind described in the antecedent may come to pass. (8.192)

Such, then, Peirce declares, ought to be our conception of law.

In a very early paper ( $c a$. 1866) Peirce discussed the question whether in a world of chance all law would be abrogated. His opinion was that they would not, because there are two kinds of law, formal and material.

Suppose that in throwing the die other numbers had turned up from those which actually turned up, so that the row of numbers would have been somewhat different; still the laws would have held; they would hold with one set of numbers as well as with another. Whereas if we were to give a whale legs or a woman wings, the laws of the animal kingdom would be interfered with. So that there are two kinds of laws, those which in a different state of things would continue to hold good and those which in a different state of things would not hold good. The former we call formal laws, the latter material laws. (7.137)

Peirce gives the principle of induction as an example of a formal law, namely, as is the sample so is the whole and the sameness of a number of characters manifests identity of objects (7.137, cf. 7.131).

But so long as there are any laws whatsoever, these laws ... must exist. . . . Now all law may, in one sense, be contingent. But that there should be knowledge without the existence of law, that there should be intelligence without anything being intelligible, all admit to be impossible. These laws therefore cannot be abrogated without abrogating knowledge; and thus are the formal conditions of all knowledge. (7.138)

The laws of logic, therefore, including the laws of probability, are universal laws that do not depend for their validity upon the peculiarities of the world in which we happen to live. They would hold good in any world whatsoever that was knowable. Even a world of pure chance, if it were at all knowable, would be subject to these 
laws of reason. This notion of formal law, law of reason, plays an important role in Peirce's theory of tychism. The world in which we live is not a world of pure chance nor is it a world of absolute determinism. As a matter of fact the material laws of our world admit of variation, exception, and growth, which can be accounted for only by admitting that chance is a real principle in its constitution. Nevertheless, in a world of chance and of law Peirce will hold that there is a destiny-something to be fulfilled: the growth of concrete reasonableness. This growth requires formal laws of reason which give it direction and stability amid the variation and vagaries of particular material laws.

Forty years later, Peirce described the third mode of reality as that which was destined or sure to come true (4.547), and he explained that "destined" meant that which is sure to come "although there is no necessitating reason for it" $(4.547, \mathrm{n} .1)$. The example he uses is that if a pair of dice are thrown often enough, they will be sure to turn up sixes some time, although there is no necessity that they should. What assures us that they will surely turn up sixes sometime or other is the law of probability. Indeed, the probability that they will so behave in the long run is one. And yet there is no necessitating cause that they should. The throw of the dice follow the formal law of probability, although there is no given set of circumstances which determine just when the sixes will appear. Still as a matter of fact there are no formal laws really at work except in some sort of universe with its material laws. Indeed we believe that Peirce held that it is the formal laws that not only allow us to discover the material laws of our universe at any particular time but also make those laws to be laws. It is the formal laws of reason that create in the world regularities, potentialities, habits of uniform activity ( subject, of course, to modification and growth through the operation of chance variations which may tend to establish new regularities ). ${ }^{13}$ There is evidence for such an interpretation in the following portion of the paragraph under consideration.

I do not see by what confusion of thought anybody can

13 Hence Peirce's remark to Calderoni that classification of the elements of thought and consciousness according to their formal structure is what is important. This he tried to do in his categories (8.213). 
persuade himself that he does not believe that tomorrow is destined to come. The point is that it is today really true that tomorrow the sun will rise; or that, even if it does not, the clocks or something, will go on. For if it be not real it can only be fiction: a Proposition is either True or False. But we are too apt to confound destiny with the impossibility of the opposite. I see no impossibility in the sudden stoppage of everything. In order to show the difference, I remind you that "impossibility" is that which, for example, describes the mode of falsity of the idea that there should be a collection of objects so multitudinous that there would not be characters enough in the universe of characters to distinguish all those things from one another. Is there anything of that sort about the stoppage of all motion? There is, perhaps, a law of nature against it; but that is all. (4.547)

Clearly Peirce is trying to distinguish "destiny" from "the impossibility of the opposite." He asks us to consider two cases: (1) the sudden stoppage of all motion, and (2) a greatest multitude of actual objects. These cases cannot be called "impossible" in precisely the same sense. Case 2 is logically impossible because it involves a contradiction. ${ }^{14}$ In other words, it is strictly unthinkable because it violates a formal law of intelligibility. Case 1 may be physically impossible given the structure of our world, that is, there may be a "law of nature" against it, but it is not strictly unthinkable since it does not seem to involve any contradiction in terms.

14 At least this is how we interpret that rather obscure sentence, "In order to show the difference . . . " From the context it seems certain that he is talking about logical "impossibility" because he is referring to a mode of falsity. But something is logically impossible only if it violates the principle of contradiction. The obscurity arises from the example which Peirce proposes. It has to do with the thorny issue of whether there can be a greatest multitude. Is the notion contradictory? As Murphey points out, the term "multitude" is used ambiguously for both series and collection (op. cit., p. 274). It is clear, however, that, regardless of whether Peirce's proof of his paradox is correct or not, he meant to show a contradiction and hence an example of the strictly "impossible." See ibid., 238-288, for an excellent discussion of the issue involved. 
Now, Peirce wants to say that the indefinite continuation of motion may be destined even though its opposite does not involve a contradiction. Neither the continuation of motion indefinitely, nor the sudden stoppage of everything is unthinkable. Either might serve as an hypothesis which must be supported by observation and analysis of experience. Neither can be ruled out a priori. On the other hand, we can infer that a "greatest multitude" of actual objects could not be destined since it is contradictory. Whatever be the constitution of our world, a priori knowledge indicates at least one characteristic it could not have.

Regardless of how apt one might judge Peirce's examples to be, the point he is making is clear enough and bears out our discussion concerning formal and material laws. To summarize what can be deduced from this passage: (1) for Peirce the "impossibility of the opposite" means "what involves a contradiction;" (2) since the principle of contradiction is a logical law, the sort of possibility Peirce here has in mind is "logical" (logical possibility is a necessary, but not sufficient, condition for real potentiality); (3) the formal or logical law of contradiction does not afford us any positive evidence of what the real potentialities of our world are, but rather sets a negative boundary to them; (4) therefore, the laws of nature or material laws must be discovered by inductive inference; (5) these alone are the basis for our beliefs about future occurrences; (6) the logical possibility of these laws being contravened does not alter the fact that the evidence on hand that they will not be so contravened may give us a probability of one. The formal laws of logic therefore are the unchanging conditions necessary for our knowledge of changeable material laws. But since, as has been shown, Peirce's synechism requires that the laws of logic also be laws of being, the formal laws of thought are not simply laws of "our mind," but laws of the intelligibility of things. They furnish, therefore, the formal principle of regularity to the world of changing fact. At any given moment our knowledge of the laws of nature (material laws) is a union of the known facts and the laws of scientific inference governing the facts. The facts might change and so the material laws, but not the laws of rationality. The change in the facts, if and when it occurs, is to be set down to real chance. Peirce's tychism will put it this way: no material law is exact, not merely because we have im- 
perfect knowledge of it, but also because of the very nature of our world. Destiny, therefore, is the inevitable working out in the world of the laws of reason without absolute determination of the facts. Destiny seems to be for Peirce something like an objectified probability theory. ${ }^{15}$ Thus the summum bonum is the growth of concrete reasonableness-the continual process of the embodiment of laws of reason in fact. ${ }^{16}$

We have remarked that Peirce frequently likens law to a general sign. Indeed, Thirdness or mediation, he says, "reaches its fullness in Representation" (5.104). Perhaps this lead will throw more light on our inquiry into the nature of law as Thirdness.

In a paper entitled, "The Regenerated Logic," published in the Monist (1896) Peirce analyses what is involved in making an assertion. Every assertion supposes a sign-maker who delivers it and some interpreter or other who will receive it. Some of the signs employed are supposed to excite in the mind of the receiver familiar images ("we might almost say, dreams"), that is, "reminiscences of sights, sounds, feelings, tastes, smells, or other sensations, now quite detached from the original circumstances of their first occurrence, so that they are free to be attached to new occasions" (3.433, emphasis added). These images themselves are signs-signs by resemblance, or icons-of the real quality (Firstness) of the thing referred to. This is the assertion's predicate (3.433). But the assertion which the deliverer seeks to convey to the receiver relates to some object or objects forced on his attention in the course of experience, and if he is to communicate he must also succeed in forcing those same objects on the receiver's attention. The icon, however, cannot do the job because it does not relate to any particular thing. Some sign like "this" or "that" or "hullo" must be used. This sort of sign is called an

15 “. . . thought, controlled by a rational experimental logic, tends to the fixation of certain opinions, equally destined, the nature of which will be the same in the end, however the perversity of thought of whole generations may cause the postponement of the ultimate fixation" (5.430).

16 Since "generals" are not only real, but active forces in the world, Peirce could write: "Accordingly, the pragmaticist ... makes it [the summum bonum] to consist in that process of evolution whereby the existent comes more and more to embody those generals which were just now said to be destined, which is what we strive to express in calling them reasonable" (5.433). 
index. An index does not describe the quality of its object; it merely points to it (Secondness, or "hecceity"). This is the assertion's subject(s) (3.434). But Peirce observed, "Neither the predicate, nor the subjects, nor both together, can make an assertion" (3.435). The assertion represents a compulsion which experience brings upon the deliverer or sign-maker to attach the predicate to the subjects in a particular way. Once this compulsion is felt, it attains a certain permanence.

This compulsion strikes him at a certain instant; and he remains under it forever after. It is, therefore, different from the temporary force which the hecceities exert upon his attention. This new compulsion may pass out of mind for the time being; but it continues just the same, and will act whenever the occasion arises, that is, whenever those particular hecceities and that first intention are called to mind together. (3.435)

As Peirce observes, this is merely the description of a permanent conditional force, or law. Therefore, to make an assertion the deliverer requires a new kind of sign distinct from the icon and the index which shall signify a law to the effect that "to objects of indices an icon appertains as a sign of them in a given way." That sort of sign is a symbol, and is the copula of the assertion (Thirdness). ${ }^{17}$

The following year (1897) in the same journal Peirce expounded the same ideas in "The Logic of Relatives." Arguing that class names should be dispensed with in favor of verbs, he writes:

A verb by itself signifies a mere dream, an imagination unattached to any particular occasion. It calls up in the mind an icon. A relative is just that, an icon, or image, without attachments to experience, without "a local habitation and a name," but with indications of the need of such attachments. (3.459)

This, of course, is unattached quality or Firstness.

An indexical word, such as a proper noun or demonstrative or selective pronoun, has force to draw the attention of the 17 Cf. also 2.249 ff., 2.293. 
listener to some hecceity common to the experience of the speaker and listener. (3.460)

This is the concrete individual or Secondness to which the quality is attached or in which it is embodied in experience.

Contrast this [the "hecceity"] with the signification of the verb, which is sometimes in my thought, sometimes in yours, and which has no other identity than the agreement between its several manifestations. That is what we call an abstraction or idea. (3.460)

This is what the nominalist would call a mere name and what the platonizing realist would say is real. According to Peirce the correct opinion requires that we strike out the nominalist's "mere" and replace the Platonist's "is" by "may be." The force of the "may-be" is this: An idea is or exists

provided experience and reason shall, as their final upshot, uphold the truth of the particular predicate, and the natural existence of the law it expresses, and this is likewise true. (3.460, emphasis added)

In other words, to go from "may be" to "is" requires the mediation of some law or conditional necessity. It is only real law which allows one to assert that such and such an icon belongs to such and such an index-or, if one prefers, that such and such a quality is embodied in such and such a "hecceity." It is the sentence or proposition which makes the assertion. It signifies or expresses a law.

The proposition, or sentence, signifies that an eternal fitness, or truth, a permanent conditional force, or law, attaches certain hecceities to certain parts of an idea. (3.461)

Ideas or abstractions, therefore, escape from being "lifeless things" by being embodied in concrete instances through real laws of nature (4.447-448). 